\title{
Teleportation: from probability distributions to quantum states
}

\author{
M. Koniorczyk ${ }^{\dagger, \ddagger}$, T. Kiss ${ }^{\dagger, \ddagger}, J^{\prime}$. Janszky $^{\dagger}$ \\ $\dagger$ Department of Nonlinear and Quantum Optics, Research \\ Institute for Solid State Physics and Optics, Hungarian Academy of \\ Sciences, P.O. Box 49, H-1525 Budapest, Hungary \\ ‡ Institute of Physics, University of Pécs, \\ Ifjúság útja 6. H-7624 Pécs, Hungary
}

November 20, 2018

\begin{abstract}
The role of the off-diagonal density matrix elements of the entangled pair is investigated in quantum teleportation of a qubit. The dependence between them and the off-diagonal elements of the teleported density matrix is shown to be linear. In this way the ideal quantum teleportation is related to an entirely classical communication protocol: the one-time pad cypher. The latter can be regarded as the classical counterpart of Bennett's quantum teleportation scheme. The quantum-to-classical transition is demonstrated on the statistics of a gedankenexperiment.
\end{abstract}

PACS Nubmers: 03.67.Hk, 03.65.Bz

\section{Introduction}

The quantum teleportation phenomenon [1] has become one of the central aspects in the investigations of quantum entanglement. Its possible applicability for quantum communication and its experimental realization in different physical systems [2, 3, 因, 可 gave rise to a wide spread interest in several 
related problems, including possible alternative schemes [6, 0, 8, 9, 10], discussion and resolution of experimental and theoretical limitations [11, 12, 13] and the connection to nonlocality and inseparability [14, 15, 16].

The relation of nonlocality and inseparability of mixed states has already been a challenging problem before the advent of quantum teleportation. Werner [17] introduced inseparable mixed states which do not violate Bell's inequalities. Several measures have been defined to quantify the amount of entanglement contained in mixed states [18, 19, 20, 21], partly inspired by entanglement distillation protocols [22, 23, 24, 25]. Whether it is possible to employ a bipartite mixed state for quantum teleportation has turned out to be an additional nontrivial question in this field [14, 26, 27]. Recently, Bose and Vedral [28] have investigated the direct relation between the mixedness of a state and its usefulness for teleportation. They applied the von Neumann entropy $S(\varrho)=-\operatorname{Tr}(\varrho \ln \varrho)$ as a measure for mixedness, and find that there is a threshold for this quantity above which the bipartite state $\varrho$ becomes useless for teleportation.

In order to separate the quantum and classical aspects of communication, N. and B. Gisin [29] and independently Steiner [30] have presented a local hidden variable (LHV) model which, supplied by classical communication, is capable of reproducing quantum correlations of two spin $1 / 2$ particles in singlet state. Brassard et al. 31 quantify the amount of classical communication required to be supplemented to simulate quantum correlations for given numbers of qubits. More recently, Cerf et al. 32 have presented a protocol in an LHV model for teleporting a quantum state via classical communication. On the other hand, M. Żukowski [33] has investigated the connection of local realism and the nonclassical part quantum teleportation process, and concluded, that no local hidden variable model can reproduce the quantum prediction.

The effect of mixedness of a bipartite state applied for teleportation is obviously an important aspect in understanding the classical-quantum limit. The classical limit of the original protocol of Bennett has not yet been investigated. Quantum teleportation, the basic primitive of quantum communication networks consists of the transmission of a quantum state via a combination of classical channels and quantum correlations. In lack of either the classical channel or the entangled resource, only noise is obtained. But what is the classical analogue of Bennett's scheme? To answer this question, we investigate a set of bipartite states interpolating between an ideal entangled state and a possible classical limit. This approach provides a transition 
from the quantum teleportation to its classical counterpart.

In this paper we examine, what happens to a teleportation scheme, when the density matrix elements of the entangled state used as a correlated pair, which are off-diagonal on product basis, are reduced. This approach, turning the entangled state into a classical correlation, obviously offers a possible way of obtaining a classical limit of the teleportation process.

The paper is organized as follows: in section 2 the class of bipartite states in argument is described, and Bennett's protocol of quantum teleportation of a qubit is summarized in a consistent density matrix formalism. The latter can be regarded as a special case of the treatment of e. g. [34 or [16]. By replacing the ideal EPR pair with the states investigated, we obtain our main result. Starting from this, two examples are studied in detail: in section 3 the purely classical limit is introduced, and in section 4 cases between the ideal quantum teleportation and the classical limit are analyzed by examining a gedankenexperiment. Section 5 summarizes the results.

\section{Quantum teleportation revisited}

We restrict ourselves to two-level systems, and use the terminology of spin- $\frac{1}{2}$ particles. The results can be applied to any representation of qubits, e. g. for the polarization of single photon states, for which quantum teleportation is experimentally feasible [2]. The spin $z$-component eigenstates of the particles are denoted by $|\uparrow\rangle$ and $|\downarrow\rangle$. Density matrices of the bipartite systems, if not otherwise stated, are represented on the natural product state basis $\{|\uparrow\rangle|\uparrow\rangle,|\uparrow\rangle|\downarrow\rangle,|\downarrow\rangle|\uparrow\rangle,|\downarrow\rangle|\downarrow\rangle\}$. We will also use the notation

$$
\begin{aligned}
\left|\Psi^{( \pm)}\right\rangle & =\frac{1}{\sqrt{2}}(|\uparrow\rangle|\downarrow\rangle \pm|\downarrow\rangle|\uparrow\rangle) \\
\left|\Phi^{( \pm)}\right\rangle & =\frac{1}{\sqrt{2}}(|\uparrow\rangle|\uparrow\rangle \pm|\downarrow\rangle|\downarrow\rangle)
\end{aligned}
$$

for the four Bell-states.

Let us examine Bennett's scheme of quantum teleportation [1] of a qubit: the sender, Alice has particle 1 of $\frac{1}{2}$ spin in the state

$$
\varrho_{\text {in }}^{(1)}=\left(\begin{array}{ll}
\varrho_{00} & \varrho_{01} \\
\varrho_{10} & \varrho_{11}
\end{array}\right),
$$

and wants to teleport it to Bob. The upper indices of density matrices (and other operators) refer to the number assigned to the particles. They share 
particles 2 and 3 in a state $\varrho^{(23)}$ as an entangled resource, and there is also a classical communication channel between them. Alice has an ideal Bell-state detector, and Bob can carry out unitary transformations on particle 3 given to him. As a starting point of our investigation, let us suppose, that the state of particles 2 and 3 is described by the following density matrix:

$$
\begin{array}{r}
\varrho_{\text {shared }}^{(23)}(\alpha)=\frac{1}{2}\left(\left|\uparrow_{2}\right\rangle\left|\downarrow_{3}\right\rangle\left\langle\uparrow_{2}\left|\left\langle\downarrow_{3}|+| \downarrow_{2}\right\rangle\right| \uparrow_{3}\right\rangle\left\langle\downarrow_{2}\right|\left\langle\uparrow_{3}\right|\right. \\
\left.-\alpha\left|\uparrow_{2}\right\rangle\left|\downarrow_{3}\right\rangle\left\langle\downarrow_{2}\left|\left\langle\uparrow_{3}|-\alpha| \downarrow_{2}\right\rangle\right| \uparrow_{3}\right\rangle\left\langle\uparrow_{2}\right|\left\langle\downarrow_{3}\right|\right) .
\end{array}
$$

The lower indices in the kets indicate the number assigned to the particles. Let $\alpha$ be real parameter between 0 and 1 . For $\alpha=1, \varrho_{\text {shared }}^{(23)}(1)=$ $\left|\Psi_{23}^{(-)}\right\rangle\left\langle\Psi_{23}^{(-)}\right|$, thus in this case Alice and Bob share an EPR pair. This is the case of ideal quantum teleportation. Otherwise, the off-diagonal elements of the density matrix are multiplied by $\alpha$. For $\alpha=0$ the density matrix is diagonal in the product state basis, describing classical statistics only. This state is the mixture of the product states $\left|\uparrow_{2}\right\rangle\left|\downarrow_{3}\right\rangle$ and $\left|\downarrow_{2}\right\rangle\left|\uparrow_{3}\right\rangle$. Such a state can be generated by a classically stochastic source emitting particles with opposite spins, with equal probability of sending "up" and "down" state both for particles 2 and 3 .

The states in equation (3) may be rewritten in the Bell-basis, we find

$$
\varrho_{\text {shared }}^{(23)}(\alpha)=\frac{1+\alpha}{2}\left|\Psi_{23}^{(-)}\right\rangle\left\langle\Psi_{23}^{(-)}\left|+\frac{1-\alpha}{2}\right| \Psi_{23}^{(+)}\right\rangle\left\langle\Psi_{23}^{(+)}\right|,
$$

that is, the state is a mixture of the two $\Psi$ Bell-states. The class of states examined here is a subclass of Werner states (which were examined from teleportation's point of view in references [15, 28]) where the two $\Phi$ states do not occur.

Let us consider now the entire teleportation process. Initially, the state of the whole system of the three particles is the product of the states in Eqs. (2) and (3):

$$
\varrho_{\text {in }}^{(123)}=\varrho_{\text {in }}^{(1)} \otimes \varrho_{\text {shared }}^{(23)}(\alpha) .
$$

Alice carries out a Bell-state measurement, which is described by one of the operators including projection of subsystems 1 and 2 to a Bell-state,

$$
\hat{P}_{i}^{(123)}=4\left(\left|\Psi_{i}^{(12)}\right\rangle\left\langle\Psi_{i}^{(12)}\right| \otimes \mathrm{id}^{(3)}\right),
$$

where $\left|\Psi_{i}\right\rangle$ stands for one of the four Bell-states, and $\mathrm{id}^{(3)}$ is the identity operator for the Hilbert-space of particle 3. The result of the measurement 
is $i$, corresponding to the $i$-th Bell-state. This information is sent to Bob via the classical channel. Because the detection of each Bell-state occurs with probability $\frac{1}{4}$, the operator is multiplied by 4 in order to preserve the norm of the state obtained. The state of the system after the measurement is given by applying the operator in equation (6) to the state in equation (5). From this we obtain the state of Bob's particle by tracing out in the other two particles:

$$
\varrho_{\mathrm{u}}^{(3)}=\operatorname{Tr}_{12}\left(P_{i}^{(123)} \varrho_{\mathrm{in}}^{(123)} P_{i}^{(123) \dagger}\right) .
$$

In the last step, Bob has to apply a unitary transformation $U^{(3)}$ on state $\varrho_{u}^{(3)}$, according to Alice's measurement result $i$, which is the identity operator in case Alice has detected $\left|\Psi^{(-)}\right\rangle$, and

$$
U^{(3)}=\left(\begin{array}{cc}
1 & 0 \\
0 & -1
\end{array}\right),\left(\begin{array}{ll}
0 & 1 \\
1 & 0
\end{array}\right),\left(\begin{array}{cc}
0 & i \\
-i & 0
\end{array}\right)
$$

for detecting $\left|\Psi^{(+)}\right\rangle,\left|\Phi^{(-)}\right\rangle$, and $\left|\Phi^{(+)}\right\rangle$respectively. Carrying out the calculations described, we obtain our main result: the state teleported to Bob reads

$$
\varrho_{\text {out }}^{(3)}=\left(\begin{array}{cc}
\varrho_{00} & \alpha \varrho_{01} \\
\alpha \varrho_{10} & \varrho_{11}
\end{array}\right) .
$$

It can be seen that the reduction of the off-diagonal elements of the density matrix describing the entangled resource is inherited by the teleported state. Thus teleportation acts as a phase-damping channel.

\section{The one-time-pad as a classical limit of tele- portation}

As a first example, let us examine the case of "teleporting" a classical bit. Assume, that $\alpha=0$, that is the density matrix describing the entangled resource is diagonal. According to (9), only the diagonal matrix elements of the density matrix of the input state, i.e. the statistics of the measurement of the spin- $z$ component is preserved. Therefore, let us suppose, that the input state in equation (2) is already diagonal: $\varrho_{10}=\varrho_{01}=0$. In this section we consider measurement of the $z$ components of the spins.

Under these circumstances our particles can be exactly identified with classical bits. The states of these classical bits, denoted by $\uparrow$, and $\downarrow$, are identical with the basis quantum states $|\uparrow\rangle$ and $|\downarrow\rangle$. The diagonal density 
matrix of the input quantum state describes a classical probability distribution of bit 1 . This is transferred into bit 3 via a classical communication channel and a classical correlation. The process itself can be interpreted in the following way: The source of bits 2 and 3 broadcasts correlated bit-pairs $\downarrow_{2} \uparrow_{3}$ or $\uparrow_{2} \downarrow_{3}$ with equal probability. This can be regarded as the classical limit of an EPR-pair. Bit 2 is obtained by Alice, who makes a measurement, which tells whether bit 1 and 2 are the same or different. One cannot speak of superpositions in this classical context, and therefore the two $\Psi$ and the two $\Phi$ Bell-states coincide in this limit: the former two mean simply "the two bits are different" ( $\Psi$-detection), and the latter "the two bits are the same" ( $\Phi$-detection). Thus the Bell-state measurement degenerates to an "exclusive or" operation, resulting in a single bit of information, communicated to Bob. Bob has to carry out the proper transformation to regain the "teleported" bit. There is no phase of the probability amplitudes for classical probability, thus the transformations in equation (8) degenerate to a conditional NOT operation: in case of " $\Psi$ detection", Bob has obtained bit 3 in the proper state, while in case of " $\Phi$ detection" he has to invert bit 3 . Finally bit 3 is left with the original value of bit 1 . Since the values of bits 1 or 2 are irrelevant from the "Bell-state measurement", it is not necessary for Alice to be aware of the actual value of bit 1 to be "teleported". Therefore the method works for "teleporting" an unknown classical bit as well, similarly to the quantum protocol.

The classical protocol described here is well known as the one-time-pad in classical cryptography [35]. This is, in some sense, the classical protocol most similar to quantum teleportation. It is the "teleportation" of the (optionally unknown) state of a classical bit via a classical communication channel and a classical correlation. The classical correlation provides noise in the lack of the classical communication channel, while the classical channel itself is useless for reconstruction of the result without the correlation. Note that instead of measuring the state of bit 1 and simply communicating its value, only a comparison with a reference has been made.

\section{Statistics of a gedankenexperiment}

Having described the classical analogue, we may now examine $\alpha \neq 0$, which interpolates between the classical and the quantum case. We calculate the consequence of (9) to the result of a teleportation experiment. For simplicity, 
let us suppose, that we want to teleport the state $|\uparrow\rangle$, having $z$-component of $+\frac{1}{2}$, rotated by a given angle $\phi$ around the $x$ axis of the coordinate system. The operator of this rotation $(\hbar=1)$ is

$$
\hat{R}(\phi)=e^{\frac{i}{2} \phi \sigma_{x}}
$$

$\sigma_{x}$ being the first Pauli-matrix, and thus we have the state

$$
\left|\Psi_{\text {in }}(\phi)\right\rangle=\hat{R}(\phi)|\uparrow\rangle=\left(\begin{array}{c}
\cos \left(\frac{\phi}{2}\right) \\
i \sin \left(\frac{\phi}{2}\right)
\end{array}\right)
$$

to be teleported. According to (9), we obtain

$$
\varrho_{\text {out }}(\phi, \alpha)=\left(\begin{array}{cc}
\cos \left(\frac{\phi}{2}\right)^{2} & -i \alpha \cos \left(\frac{\phi}{2}\right) \sin \left(\frac{\phi}{2}\right) \\
i \alpha \cos \left(\frac{\phi}{2}\right) \sin \left(\frac{\phi}{2}\right) & \sin \left(\frac{\phi}{2}\right)^{2}
\end{array}\right)
$$

as result of the teleportation process. In order to verify the teleportation, one may measure the spin along an axis obtained by rotating the $z$ axis with the angle $\phi_{m}$ around the $\mathrm{x}$ axis. The probability of finding this component of the spin $+\frac{1}{2}$ is in this case is

$$
\begin{array}{r}
\mathcal{P}\left(\phi, \phi_{m}, \alpha\right)=\operatorname{Tr}\left(\varrho_{\text {out }}(\phi, \alpha) R\left(\phi_{m}\right)|\uparrow\rangle\langle\uparrow| R^{\dagger}\left(\phi_{m}\right)\right) \\
=\frac{\cos (\phi) \cos \left(\phi_{m}\right)}{2}+\frac{\alpha \sin (\phi) \sin \left(\phi_{m}\right)}{2}+1 / 2 .
\end{array}
$$

For $\alpha=1$ we obtain the familiar cosine-type result valid for ideal teleportation,

$$
\mathcal{P}\left(\phi, \phi_{m}, 1\right)=\frac{\cos \left(\phi-\phi_{m}\right)+1}{2},
$$

which is equal to 1 for $\phi=\phi_{m}$, meaning perfect teleportation to any direction. For a given input state $\left|\Psi_{\text {in }}(\phi)\right\rangle$, the probability for finding the output in the input state after the teleportation is the fidelity of the teleportation of the input state. This fidelity is

$$
\mathcal{P}(\phi, \phi, \alpha)=-\frac{1}{4}[\alpha(\cos 2 \phi-1)-\cos 2 \phi-3] .
$$

In figure 1 we have plotted this function. It is equal to 1 in the case of ideal teleportation. The basis states $|\uparrow\rangle$ and $|\downarrow\rangle$ are always properly teleported, as we have seen in the classical case. The minimum of the fidelity for any $\alpha$ 


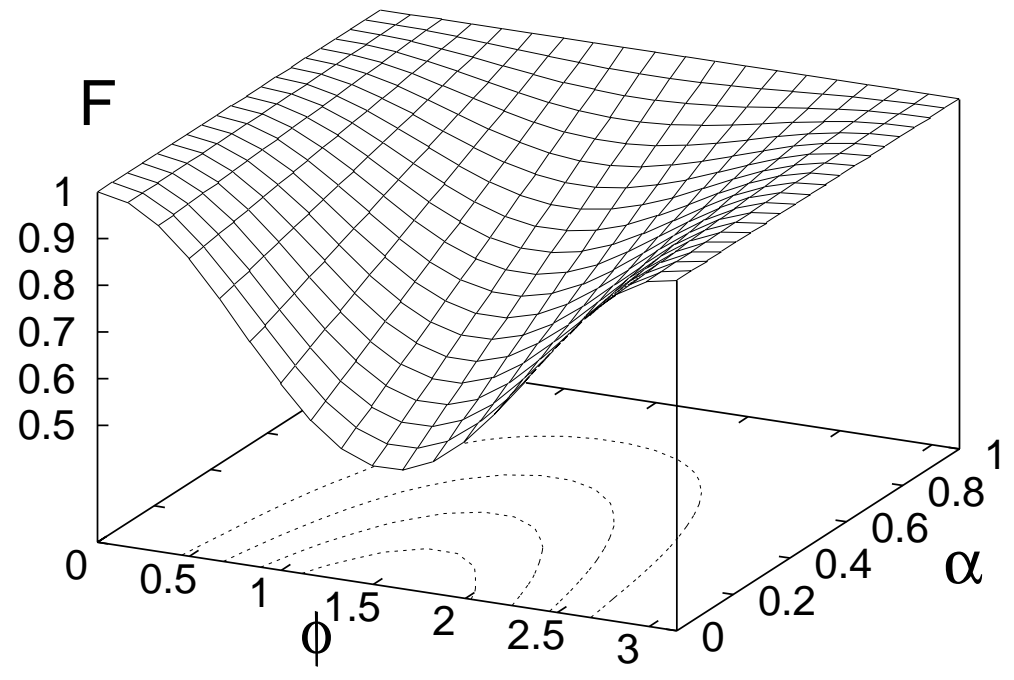

Figure 1: The fidelity of the teleportation of the spin pointing to the direction described by the angle $\phi$ plotted against the parameter $\alpha$ describing the impurity of the state.

occurs for the equal superposition of the two basis states $\left(\phi_{m}=\pi / 2\right)$. The minimum value for $\alpha=0$ is $\frac{1}{2}$, expressing that the result of the measurement can be either $\uparrow$ or $\downarrow$ with equal probability, thus this state is not at all teleported. Increasing the purity of the mixed state increases the domain of the angles in which teleportation can be regarded as reliable.

\section{Conclusion}

We have studied the role of the off-diagonal density matrix elements of the entangled pair in the teleportation of a qubit. By setting these to zero, we have obtained the classical one-time pad cypher, as a classical limit of quantum teleportation. We have demonstrated the result on the behavior of statistics and fidelity of a gedankenexperiment. We have found, that the 
fidelity of the teleportation is most sensitive to the loss of the quantum entanglement in the EPR pair for a state at a right angle to the quantization direction.

\section{Acknowledgements}

This work was supported by the Research Fund of Hungary (OTKA) under contracts Nos. T023777, T034484 and F032346. T. K. acknowledges the support of the Hungarian Academy of Sciences (Bolyai János Kutatási Ösztöndíj).

\section{References}

\section{References}

[1] Bennett C. H, Brassard G, Crépeau C, Jozsa R, Peres A and Wootters W. K 1993 Phys. Rev. Lett. 701895

[2] Bouwmeester D, Pan J.-W, Mattle K, Eibl M, Weinfurter H and Zeilinger A 1997 Nature 390575

[3] Boschi D, Branca S, Martini F. D, Hardy L and Popescu S 1998 Phys. Rev. Lett. 801121

[4] Furusawa A, Sorensen J. L, Braunstein S. L, Fuchs C. A, Kimble H. J and Polzik E. S 1998 Science $\mathbf{2 8 2} 706$

[5] Nielsen M. A, Knill E and Laflamme R 1998 Nature 39652

[6] Villas-Bôas C. J, de Almeida N. G and Moussa M. H. Y 1999 Phys. Rev. A 602759

[7] Stenholm S and Bardroff P. J 1998 Phys. Rev. A 584373

[8] Koniorczyk M, Kurucz Z, Gábris A and Janszky J 2000 Phys. Rev. A 62013802

[9] Braunstein S. L, D'Ariano G. M, Milburn G. J and Sacchi M. F 2000 Phys. Rev. Lett. 843486 
[10] D'Ariano G. M, Presti P. L and Sacchi M. F 2000 Phys. Lett. A 27232

[11] Paris M. G. A, Plenio M. B, Bose S, Jonathan D and D'Ariano G. M 2000 Phys. Lett. A 273153

[12] Lütkenhaus N, Calsamiglia J and Suominen K. A 1999 Phys. Rev. A 59 3295

[13] Kok P and Braunstein S 2000 Phys. Rev. A 61042304

[14] Popescu S 1994 Phys. Rev. Lett. 72797

[15] Gisin N 1996 Phys. Lett. A 210157

[16] Horodecki M, Horodecki P and Horodecki R 1999 Phys. Rev. A 601888

[17] Werner R. F 1989 Phys. Rev. A 404277

[18] Vedral V and Plenio M. B 1998 Phys. Rev. A 571619

[19] Wootters W. K 1998 Phys. Rev. Lett. 802245

[20] Bennett C. H, DiVincenzo D. P, Smolin J. A and Wootters W. K 1996 Phys. Rev. A 543824

[21] Vidal G and Tarrach R 1999 Phys. Rev. A 59141

[22] Bennett C. H, Bernstein H. J, Popescu S and and B. S 1996 Phys. Rev. A 532046

[23] Gisin N 1996 Phys. Lett. A 210151

[24] Bennett C. H, Brassard G, Popescu S, Schumacher B, Smolin J. A and Wootters W. K 1996 Phys. Rev. Lett. 76722

[25] Parker S, Bose S and Plenio M. B 2000 Phys. Rev. A 61032305

[26] Fisher D. G, Mack H and Freyberger M 2000 e-print, quant-ph/0008038

[27] Lee J, Kim M. S and Yeong H 2000 Phys. Rev. A 62032305

[28] Bose S and Vedral V 2000 Phys. Rev. A 61 040101(R)

[29] Gisin N and Gisin B 1999 Phys. Lett. A 260323 
[30] Steiner M 1999 e-print, quant-ph/9902014

[31] Brassard G, Cleve R and Tapp A 1999 Phys. Rev. Lett. 831874

[32] Cerf N. J, Gisin N and Massar S 2000 Phys. Rev. Lett. 842521

[33] Żukowski M 2000 Phys. Rev. A 62032101

[34] Nielsen M and Caves C. M 1997 Phys. Rev. A 552547

[35] Schneier B Applied Cryptography 2nd ed. (John Wiley \& Sons, New York, 1996) 